Revista Perspectivas Online: Biológicas \& Saúde Junho/2020, v.10, n.33, p.1- 14

ISSN: 2236-8868 (Online)

DOI: $10.25242 / 8868103320201990$

\title{
PERFIL DOS CASAIS INFÉRTEIS SUBMETIDOS À TÉCNICA DE REPRODUÇÃO HUMANA ASSISTIDA
}

\author{
Deborah Costa de Jesus dos Santos ${ }^{1}$, Felipe dos Santos Fraga ${ }^{1}$ \& Iukary Takenami ${ }^{2 *}$
}

\section{RESUMO}

SANTOS, D.C.J.; FRAGA, F.S.; \& TAKENAMI, I. Perfil dos casais inférteis submetidos à técnica de reprodução humana assistida. Perspectivas Online: Biológicas \& Saúde, v.10, n.33, p.1-14, 2020.

O objetivo deste estudo foi caracterizar o perfil dos casais inférteis submetidos à técnica de reprodução humana assistida (RHA) no município de Salvador, Bahia. Trata-se de um estudo de caráter retrospectivo, observacional, baseado em dados secundários, obtidos mediante consulta aos prontuários eletrônicos dos pacientes cadastrados em uma clínica particular de RHA durante o ano de 2017. As variáveis categóricas e quantitativas foram apresentadas em valor absoluto e relativo, média \pm desvio-padrão, respectivamente. Posteriormente, analisadas por meio de um software estatístico. Dos 496 pacientes atendidos na clínica de reprodução, $360(72,6 \%)$ foram diagnosticados com infertilidade. A causa compartilhada $(n=224 ; 62,2 \%)$ foi mais prevalente do que a isolada por sexo. Entre as mulheres, observa-se que a idade avançada $(30,8 \%)$ e endometriose $(26,5 \%)$ representam as principais causas de infertilidade. Nos homens, a teratozoospermia $(36,9 \%)$ e a varicocele
$(33,6 \%)$ foram os fatores que mais comprometeram a fertilidade. A qualidade do gameta feminino e masculino é severamente comprometida em pacientes que tem como causa de infertilidade a falência ovariana $(62,5 \%) \quad \mathrm{e}$ oligoastenoteratozoospermia $\quad(56,5 \%)$, respectivamente. No entanto, observa-se que nas mulheres, a baixa qualidade embrionária está mais associada à obstrução tubária $(47,7 \%)$, enquanto que nos homens, o mesmo fator associado a baixa qualidade seminal é também responsável pelo desenvolvimento insatisfatório dos embriões $(69,2 \%)$. Por fim, conclui-se que a infertilidade acomete, em maior proporção, homens e mulheres simultaneamente. Nas mulheres, a infertilidade associa-se a idade avançada e endometriose, enquanto que nos homens a teratozoospermia e varicocele. Essas causas, além de impactarem na qualidade dos gametas, também afetam a qualidade e desenvolvimento embrionário.

Palavras-chave: Infertilidade; Feminina; Masculina; Reprodução Humana Assistida. 


\title{
PROFILE OF INFERTILE COUPLES SUBMITTED TO ASSISTED HUMAN \\ REPRODUCTION TECHNIQUE
}

\begin{abstract}
The aim of this study was to characterize the profile of infertile couples submitted to assisted human reproduction (AHR) treatment in the municipality of Salvador, Bahia. This is a retrospective, observational study based on secondary data obtained by analyzing the electronic medical records of patients enrolled in the laboratory of a private assisted reproduction clinic during the period of 2017. The categorical and quantitative variables were presented in absolute value and relative, mean \pm standard deviation, respectively. Subsequently, analyzed using a statistical software. Of the 496 patients attended in the clinic reproduction, 360 $(n=360 ; 72.6 \%)$ were diagnosis with infertility. The shared cause $(n=224$; $62.2 \%$ ) was more prevalent than the one isolated by sex. The most common causes of infertility observed in women were advanced age $(30.8 \%)$ and endometriosis

varicocele $(33.6 \%)$ were the factors that most compromised male fertility. The quality of the female and male gamete is severely compromised in volunteers whose infertility causes ovarian failure $(62.5 \%)$ and oligoastenoteratozoospermia $(56.5 \%)$, respectively. However, it is observed that in women the low embryo quality is more associated with tubal obstruction (47.7\%), while in men, the same factor associated with poor seminal quality is also responsible for the unsatisfactory development of embryos (69.2\%). Finally, it is concluded that infertility affects, in greater proportion, men and women simultaneously. In women, infertility is associated with advanced age and endometriosis, while in men, teratozoospermia and varicocele. These causes, in addition to impacting the quality of gametes, also affect embryonic quality and development.
\end{abstract} $(26.5 \%)$. Teratozoospermia (36.9\%) and

Keywords: Infertility; Female; Male; Assisted Human Reproduction.

\footnotetext{
${ }^{1}$ Escola de Ciências da Saúde - Universidade Salvador - UNIFACS - Av. Luís Viana, 3100, Imbuí, Salvador, BA, CEP: 441720-200, Brasil.

${ }^{2}$ Colegiado de Medicina - Universidade Federal do Vale do São Francisco - UNIVASF - Rua da Alvorada, s/n, General Dutra, Paulo Afonso, BA, CEP: 48607-190, Brasil.

(*) e-mail: iukary.takenami@univasf.edu.br

Data de recebimento: 12/04/2020. Aceito para publicação: 31/05/2020. Data da publicação: 05/06/2020
}

Persp. online: biol. \& saúde, Campos dos Goytacazes, 33 (10) 1-14, 2020 seer.perspectivasonline.com.br 


\section{INTRODUÇÃO}

A infertilidade é definida como a incapacidade de um casal alcançar a concepção após doze meses de relações sexuais regulares sem uso de contracepção (HOCHSCHILD et al., 2009). Atualmente, essa desordem clínica é reconhecida pela Organização Mundial da Saúde (OMS) como um problema de saúde pública de grande relevância, uma vez que afeta não somente à capacidade reprodutiva do indivíduo, como também acresce significativas consequências sociais, econômicas e demográficas (OMS, 2008). Estima-se que, a nível global, 8 a 12\% dos casais tenham algum problema de infertilidade (COSTA et al., 2016).

No Brasil, mais de 278 mil casais em idade fértil apresentam alguma dificuldade em conceber um filho (GRADVOHL; OSIS; MAKUCH, 2013), o que reflete numa procura emergente por auxílio médico especializado para obtenção de uma gravidez. Segundo Queiroz (2018), durante o período de 2011 a 2016, o número de técnicas de reprodução humana assistida (RHA) realizadas no país cresceu em, aproximadamente, 150\%. O aumento na procura por tratamentos de RHA é multifatorial, envolvendo aspectos físicos, anatomofisiológicos, psíquicos e sociais (BENTO, 2014). Além disso, pode acometer a mulher, o homem, ou ambos os sexos (BABLOK et al., 2001).

Atualmente, o maior determinante da infertilidade feminina é a idade. $\mathrm{O}$ aumento da participação da mulher no mercado de trabalho impacta, como consequência, por postergar os planos de maternidade para depois dos 35 anos, época em que a fertilidade diminui significativamente, o que contribui para o envelhecimento qualitativo e a diminuição da quantidade dos oócitos liberados (GEORGE \& KAMATH, 2010). Além disso, o número de ciclos menstruais que a mulher moderna tem é muito maior, facilitando uma das doenças que mais afetam à fertilidade, a endometriose (FADHLAOUI; JOLINIÈRE; FEKI, 2014; PESSANHA et al., 2015).

Outros fatores também contribuem para o aumento da infertilidade: a difusão dos métodos contraceptivos; o início precoce da vida sexual e a maior probabilidade de ocorrência de infecções sexualmente transmissíveis derivado da multiplicidade de parceiros; o aumento do stress com repercussões no que diz respeito à função reprodutora; a obesidade; o desconhecimento dos processos reprodutivos e do período de ovulação, entre outros. Maus hábitos como ingerir bebidas alcóolicas excessivamente; fumar; má alimentação; falta ou a prática exagerada de exercícios físicos; uso inadequado de medicação e a negligência com sintomas menstruais anormais (dores constantes e mal-estar físico, menstruação irregular) também são fatores que podem influenciar na concepção (BENTO, 2014; CONG et al., 2016; DESHPANDE \& GUPTA, 2019)

Nos homens, o maior determinante de infertilidade é a qualidade do espermatozoide. Nos últimos anos, tem se observado uma queda considerável na qualidade do esperma entre indivíduos saudáveis e jovens. A etiologia para as alterações observadas na avaliação microscópica do espermatozoide pode ser atribuída, principalmente, a insuficiência testicular primária, resultante de uma anormalidade congênita ou adquirida em ambos os testículos (HARRIS et al., 2011; DURAIRAJANAYAGAM, 2018; LI et al., 2019). Além disso, hábitos cotidianos como usar roupas justas e ficar muito tempo sentado podem afetar a temperatura dos testículos e causar alterações (DURAIRAJANAYAGAM, 2018; ILACQUA et al., 2018)

Embora seja possível identificar os fatores que causam infertilidade em ambos os sexos, a prevalência de infertilidade varia consideravelmente com os diferentes critérios de avaliação diagnóstica e as diferenças regionais que ocorrem em toda extensão do território

Persp. online: biol. \& saúde, Campos dos Goytacazes, 33 (10) 1-14, 2020

seer.perspectivasonline.com.br 
brasileiro (DENTILLO, 2012). Uma vez que a infertilidade é influenciada por diversos fatores, a prevalência real desta condição é extremamente difícil de estimar. Neste cenário, ressalta-se a importância dos estudos que avaliem a prevalência de infertilidade e as características clínicas dos pacientes, as quais contribuem para a manutenção dos casos na região. Assim, a descrição e interpretação dos dados podem auxiliar e direcionar estratégias mais eficazes na prevenção da infertilidade, enquanto problema de saúde pública. Diante do exposto, o objetivo desse estudo foi caracterizar o perfil dos casais inférteis submetidos à técnica de RHA no município de Salvador, Bahia.

\section{METODOLOGIA}

Trata-se de um estudo observacional descritivo, retrospectivo, baseado em dados secundários, obtidos mediante consulta aos prontuários eletrônicos dos pacientes cadastrados no laboratório de uma clínica particular de RHA, localizado no município de Salvador, Bahia. $\mathrm{O}$ centro de reprodução é um grupo líder em medicina reprodutiva que oferece técnicas avançadas e tratamentos de reprodução humana no Brasil e em outros países.

Para a composição da amostra desse estudo foram selecionados os pacientes que se submeteram à técnica de injeção intracitoplasmáticas de espermatozoide (ICSI) durante o ano de 2017. Os casais que realizaram mais de um ciclo de tratamento durante o período do estudo, foram registrados apenas uma única vez. Assim, a amostra consistiu em 248 prontuários de casais atendidos na clínica de RHA. No entanto, para análises posteriores de caracterização, pacientes sem causa aparente de infertilidade foram excluídos do estudo.

O estudo foi aprovado pelo Comitê de Ética em Pesquisa da Universidade Salvador (UNIFACS) sob o número 2.125.575/17 (CAAE: 80209817.9.0000.5033). Para realização deste estudo, não foi necessária aplicação do Termo de Consentimento Livre e Esclarecido (TCLE), tendo em vista que o mesmo utilizou somente dados secundários obtidos a partir do banco de dados do laboratório da clínica de reprodução.

As variáveis de interesse foram coletadas na base de dados do programa FileMaker Pro 6 (versão para Windows 6.1, registrado em EE.UU) e transferidas para uma planilha eletrônica Microsoft Excel 2000 e, posteriormente, analisados por meio do software SPSS 19.0 (SPSS Inc., Chicago, IL, USA). Dois autores realizaram extração de dados, as seguintes variáveis foram recuperadas: idade, sexo, causas de infertilidade, qualidade dos gametas e qualidade embrionária. As variáveis quantitativas foram descritas pela média e desvio-padrão, enquanto que as variáveis categóricas foram apresentadas como valor absoluto e relativo (\%). $\mathrm{O}$ teste exato de Fisher e o teste $t$ Student foram utilizados para avaliação entre as variáveis categóricas e quantitativas, respectivamente. As diferenças foram consideradas estatisticamente significantes para valores de $\mathrm{p}<0,05$.

\section{RESULTADOS}

Durante o ano de 2017, 248 casais foram submetidos à técnica ICSI, perfazendo um total de 496 pacientes. Destes, $360(72,6 \%)$ foram diagnosticados com infertilidade. A taxa de infertilidade que afeta apenas a mulher ou homem foi de 27,5\% ( $n=99)$ e 10,3\% ( $n=37)$, respectivamente. No entanto, a infertilidade que acomete ambos os sexos, foi mais prevalente, 
representando $62,2 \%(\mathrm{n}=224)$ dos casos estudados, isto é, 112 casais foram diagnosticados como inférteis.

Diferentemente dos outros indivíduos, $136(27,4 \%)$ pacientes não tiveram causa de infertilidade detectada. Embora seus parceiros tenham sido diagnosticados com infertilidade, não se pode afirmar com precisão que estes são férteis. É de conhecimento geral na área de RHA que alguns casos não possuem uma causa de infertilidade detectada. Esta condição é definida como infertilidade idiopática, pois apesar do homem e da mulher preencherem as condições necessárias para a gravidez, ela não pode acontecer naturalmente (GRANJEIRO, 2018). Para fins de análise, estes indivíduos foram excluídos das análises seguintes. Assim, a amostra final consistiu de 360 pacientes.

Dos 360 pacientes diagnosticadas com infertilidade, observa-se que 78,6\% $(n=283)$ dos casos foram atribuídos a infertilidade primária (Tabela 1). Independente do sexo e da quantidade de causas, a frequência de infertilidade primária e secundária é semelhante $(p>0,05)$. No entanto, observa-se que pacientes com idade avançada que apresentam uma única causa como fator de infertilidade tem uma maior probabilidade de serem diagnosticados com infertilidade secundária.

Tabela 1: Características demográficas dos pacientes diagnosticados com infertilidade em uma clínica particular de reprodução humana assistida em Salvador, Bahia, durante o ano de $2017(\mathrm{~N}=360)$.

\begin{tabular}{|c|c|c|c|c|}
\hline \multirow[b]{2}{*}{ Características } & \multicolumn{3}{|c|}{ Infertilidade, $\mathrm{n}(\%)$} & \multirow[b]{2}{*}{$\begin{array}{c}\text { Total } \\
(\mathbf{N}=360)\end{array}$} \\
\hline & $\begin{array}{c}\text { Primária } \\
(\mathrm{N}=283)\end{array}$ & $\begin{array}{c}\text { Secundária } \\
(\mathrm{N}=77)\end{array}$ & p-valor & \\
\hline Média de idade \pm DP & $38,8 \pm 5,2$ & $42,4 \pm 6,3$ & $<0,0001 *$ & $39,6 \pm 5,6$ \\
\hline \multicolumn{5}{|l|}{ Sexo } \\
\hline Masculino & $114(40,3)$ & $35(23,5)$ & $0,432 * *$ & $149(41,4)$ \\
\hline Feminino & $169(59,7)$ & $42(19,4)$ & & $211(58,6)$ \\
\hline \multicolumn{5}{|c|}{ Quantidade de causas de infertilidade } \\
\hline 1 causa & $209(73,9)$ & $65(84,4)$ & $0,07 * *$ & $274(76,1)$ \\
\hline$\geq 2$ causas & $74(26,1)$ & $12(15,6)$ & & $86(23,9)$ \\
\hline
\end{tabular}

* Test T Student. ** Teste Exato de Fisher. DP = Desvio-padrão.

Quando estratificada por sexo, das 211 mulheres avaliadas, $167(79,1 \%)$ apresentaram apenas uma única causa e $44(20,9 \%)$ apresentaram mais de uma causa associada à infertilidade. Independentemente da quantidade de causas, as principais causas de infertilidade relatadas são apresentadas na Tabela 2. Idade avançada ( $\mathrm{n}=65,30,8 \%)$, endometriose $(n=56,26,5 \%)$ e obstrução tubária $(n=37,17,5 \%)$ foram as principais etiologias associadas às mulheres inférteis. Por outro lado, observa-se que a endometriose associada com a idade avançada $(n=7,15,9 \%)$ ou com falência ovariana $(n=7,15,9 \%)$ representam as principais causas combinadas que causam infertilidade.

Dos 149 homens avaliados, 107 (71,8\%) apresentaram apenas uma única causa e 42 $(28,2 \%)$ apresentaram mais de uma causa associada à infertilidade. As principais causas de infertilidade relatadas são apresentadas na Tabela 3. Independentemente da quantidade de alterações microscópicas observadas, todas foram reportadas como uma única causa, uma vez que morfologia, vitalidade, motilidade e quantidade são fatores relacionados à espermatogênese. Teratozoospermia $(n=55,36,9 \%)$ e a varicocele $(n=50,33,6 \%)$ foram as alterações mais frequentemente encontradas nos homens inférteis. A teratozoospermia e 
varicocele $(n=13,31 \%)$ foram as principais causas combinadas que causam infertilidade nos homens.

Tabela 2: Etiologia associada à infertilidade em mulheres atendidas em uma clínica particular de reprodução humana assistida em Salvador, Bahia, durante o ano de 2017 (N=211).

\begin{tabular}{lc}
\hline Etiologia isolada $(\mathbf{N = 1 6 7 )}$ & $\mathbf{n}(\%)$ \\
Endometriose & $56(26,5)$ \\
Falência ovariana & $17(8,1)$ \\
Falência tubária & $2(0,9)$ \\
Idade avançada & $65(30,8)$ \\
Má qualidade ovocitária & $2(0,9)$ \\
Menopausa & $4(1,9)$ \\
Miomas & $2(0,9)$ \\
Obstrução tubária & $37(17,5)$ \\
Outros & $78(36,9)$ \\
Síndrome de Ovários Policísticos & $19(9)$ \\
Etiologia combinada $(\boldsymbol{N}=\mathbf{4 4})$ & \\
Endometriose e idade avançada & $7(15,9)$ \\
Endometriose e falência ovariana & $7(15,9)$ \\
Obstrução tubária e baixa reposta & $6(13,6)$ \\
Outros & $24(54,5)$ \\
\hline
\end{tabular}

Tabela 3: Etiologia associada à infertilidade em homens atendidas em uma clínica particular de reprodução humana assistida em Salvador, Bahia, durante o ano de 2017 (N=149).

\begin{tabular}{|cc}
\hline Etiologia isolada $(\mathbf{N = 1 0 7 )}$ & $\mathbf{n}(\boldsymbol{\%})$ \\
Astenozoospermia & $10(6,7)$ \\
Astenoteratozoospermia & $10(6,7)$ \\
Azoospermia & $13(8,7)$ \\
Cariótipo* & $1(0,7)$ \\
Criptoespermia & $4(2,7)$ \\
Oligozooespermia & $5(3,4)$ \\
Oligoastenozoospermia & $9(6)$ \\
Oligoastenoteratozoospermia & $23(15,4)$ \\
Oligoteratozoopermia & $6(4)$ \\
Outros & $1(0,7)$ \\
Teratozoospermia & $55(36,9)$ \\
Varicocele & $50(33,6)$ \\
Etiologia combinada $(\boldsymbol{N}=42)$ & \\
Outros & $13(31)$ \\
Varicocele e teratozzospermia & $13(31)$ \\
Varicocele e astenoteratozoospermia & $9(21,4)$ \\
Varicocele & $7(16,7)$ \\
oligoastenoteratozoospermia & \\
*efere-se às alterações cromossômicas ocasionadas por alterações \\
$\quad$ mutagênicas, polissomias ou monossomias.
\end{tabular}

Após criteriosa avaliação pelos médicos especialistas, os casais foram submetidos à técnica de alta complexidade, ICSI. De acordo com Balaban e colaboradores (2006), os 
gametas são então classificados em bons, regulares e ruins. Durante o procedimento foram puncionados entre 1-60 (mín-máx) oócitos por paciente (total = 280 óvulos). No entanto, o resultado final da análise sobre a qualidade oocitária é representada pelo que é observado durante o procedimento de ICSI, oócito por oócito, sendo a classificação final feita com base na morfologia da maioria dos oócitos analisados por paciente. A mesma análise é feita com os gametas masculinos, no entanto, recupera-se apenas um a dois espermatozoides por paciente (total $=187$ espermatozoides). A avaliação da qualidade dos gametas recuperados é apresentada na Tabela 4.

Tabela 4: Principais causas de infertilidade feminina e masculina associadas à qualidade oocitária e seminal, respectivamente.

\begin{tabular}{lccc}
\hline \multirow{2}{*}{ Características } & \multicolumn{3}{c}{ Qualidade do gameta, n (\%) } \\
\cline { 2 - 4 } Feminina & Bom & Regular & Ruim \\
Endometriose & $8(14,3)$ & $13(23,2)$ & $35(62,5)$ \\
Falência ovariana & $4(23,5)$ & $2(11,7)$ & $11(64,8)$ \\
Falência tubária & $2(100)$ & - & - \\
Idade avançada & $12(18,5)$ & $18(27,7)$ & $35(53,8)$ \\
Menopausa & - & $1(25)$ & $3(75)$ \\
Mioma & - & $2(100)$ & - \\
Obstrução tubária & $7(19)$ & $11(29,7)$ & $19(51,3)$ \\
Outros & $17(21,8)$ & $17(21,8)$ & $44(56,4)$ \\
Síndrome de Ovário Policístico & $4(21)$ & $4(21)$ & $11(58)$ \\
Masculina & & & \\
Astenoteratozoospermia & $3(30)$ & $2(20)$ & $5(50)$ \\
Astenozoospermia & $6(60)$ & $3(30)$ & $1(10)$ \\
Azoospermia & $6(46,2)$ & $3(23,1)$ & $4(30,7)$ \\
Cariótipo* & $1(100)$ & - & - \\
Criptoespermia & $1(25)$ & - & $3(75)$ \\
Oligoastenoteratozoospermia & $8(34,8)$ & $2(8,7)$ & $13(56,5)$ \\
Oligoastenozoospermia & $4(44,5)$ & $3(33,3)$ & $2(22,2)$ \\
Oligoteratozoopermia & $3(50)$ & $1(16,7)$ & $2(33,3)$ \\
Oligozooespermia & $3(60)$ & $2(40)$ & - \\
Outros & $1(100)$ & - & - \\
Teratozoospermia & $38(69,1)$ & $13(23,6)$ & $4(7,3)$ \\
Varicocele & $32(64)$ & $9(18)$ & $9(18)$ \\
\hline Refere-se às alterações cromossômicas ocasionadas por alterações mutagênicas, polissomias ou \\
& monossomias. & & \\
& & &
\end{tabular}

Dentre os 280 oócitos recuperados, $158(56,4 \%)$ foram considerados ruins. A frequência de oócitos considerados ruins é mais elevada em pacientes diagnosticadas com falência ovariana $(62,5 \%)$, endometriose $(61,5 \%)$ e síndrome de ovário policístico $(58 \%)$, respectivamente. Embora estas sejam as principais causas associadas a obtenção de oócitos ruins, é importante ressaltar que, nenhum oócito recuperado de pacientes com menopausa ou com mioma foram considerados bons. Nos homens, apenas 43 (23\%) dos 187 foram considerados ruins. Destes, a principal causa associada a obtenção de espermatozoides ruins foram oligoastenoteratozoospermia $(56,5 \%)$ e astenoteratozoospermia (50\%). Dos quatro espermatozoides recuperados de pacientes com criptoespermia, observa-se que $75 \%$ foram considerados com qualidade seminal insatisfatória, ou seja, ruins (Tabela 4). 
Posteriormente à fecundação, a qualidade embrionária foi avaliada de acordo com os fatores de infertilidade feminina e masculina (Tabela 5). A quantidade de embriões analisados ultrapassa a quantidade de pacientes submetidos à técnica, uma vez que, para esta análise foram considerados a totalidade de embriões recuperados. Além disso, foi possível obter bons gametas provenientes de parceiros sem causa aparente de infertilidade, isso impactou diretamente na quantidade de embriões provenientes de mulheres $(\mathrm{N}=310)$ ou homens $(\mathrm{N}=251)$ e foi um limitante no estudo por conta da diferença na quantidade de embriões por casal. Na mulher, observa-se que a obstrução tubária $(47,7 \%)$, idade avançada $(44,7 \%)$ e falência ovariana $(41,7 \%)$, respectivamente, foram os fatores que mais contribuíram para a obtenção de embriões ruins ou bloqueados. Embora seja possível observar uma alta frequência de mioma, menopausa e falência tubária associados a má qualidade do embrião, deve-se avaliar estes resultados com cautela uma vez que um quantitativo pequeno de embriões foi gerado de usuárias com esse diagnóstico de infertilidade. Semelhante à qualidade seminal, o fator masculino que mais influenciou na qualidade dos embriões foram a oligoastenoteratozoospermia $(69,2 \%)$ e oligoteratozoopermia $(57,1 \%)$.

Tabela 5: Principais causas de infertilidade feminina associadas à qualidade embrionária.

\begin{tabular}{|c|c|c|c|}
\hline \multirow[b]{2}{*}{ Características } & \multicolumn{3}{|c|}{ Qualidade embrionária, n (\%) } \\
\hline & Bons & $\begin{array}{l}\text { Lentos ou } \\
\text { Regulares }\end{array}$ & $\begin{array}{c}\text { Ruins ou } \\
\text { bloqueados }\end{array}$ \\
\hline \multicolumn{4}{|l|}{ Feminina } \\
\hline Endometriose & $21(30)$ & $25(35,7)$ & $24(34,3)$ \\
\hline Falência ovariana & $6(25)$ & $8(33,3)$ & $10(41,7)$ \\
\hline Falência tubária & $1(50)$ & - & $1(50)$ \\
\hline Idade avançada & $20(23,5)$ & $27(31,8)$ & $38(44,7)$ \\
\hline Menopausa & $1(25)$ & $1(25)$ & $2(50)$ \\
\hline Mioma & $1(50)$ & 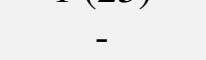 & $1(50)$ \\
\hline Obstrução tubária & $9(20,5)$ & $14(31,8)$ & $21(47,7)$ \\
\hline Outros & $22(36,1)$ & $23(37,7)$ & $16(26,2)$ \\
\hline Síndrome de Ovário Policístico & $3(16,7)$ & $8(44,4)$ & $7(38,9)$ \\
\hline \multicolumn{4}{|l|}{ Masculina } \\
\hline Astenoteratozoospermia & $5(45,5)$ & $4(36,3)$ & $2(18,2)$ \\
\hline Astenozoospermia & $6(42,8)$ & $2(14,4)$ & $6(42,8)$ \\
\hline Azoospermia & $5(29,4)$ & $4(23,5)$ & $8(47,1)$ \\
\hline Cariótipo* & $1(50)$ & $1(50)$ & - \\
\hline Criptoespermia & $2(50)$ & $1(25)$ & $1(25)$ \\
\hline Oligoastenoteratozoospermia & $5(19,3)$ & $3(11,5)$ & $18(69,2)$ \\
\hline Oligoastenozoospermia & $5(45,5)$ & $2(18,2)$ & $4(36,3)$ \\
\hline Oligoteratozoopermia & $1(14,4)$ & $2(28,6)$ & $4(57,1)$ \\
\hline Oligozooespermia & $4(50)$ & $3(37,5)$ & $1(12,5)$ \\
\hline Outros & $1(25)$ & $1(25)$ & $2(50)$ \\
\hline Teratozoospermia & $20(25,9)$ & $23(30)$ & $34(44,1)$ \\
\hline Varicocele & $17(24,3)$ & $22(31,4)$ & $31(44,3)$ \\
\hline
\end{tabular}

*Refere-se às alterações cromossômicas ocasionadas por alterações mutagênicas, polissomias ou monossomias.

\section{DISCUSSÃO}

A infertilidade é uma condição que afeta não apenas o casal proponente, mas à sociedade como um todo, uma vez que à consolidação dessa configuração familiar reduzida impactará em 
menos profissionais jovens no mercado de trabalho e risco de queda no crescimento econômico (LEITE \& FROTA, 2014). Portanto, conhecer os fatores que afetam a infertilidade é imprescindível para o estabelecimento e implantação de políticas públicas que contribuam para a diminuição de casos no município.

Um estudo realizado por Borges Jr e colaboradores (2009) demonstrou que entre janeiro de 2006 e julho de 2007, 204 casais realizaram a técnica de ICSI em uma clínica privada de fertilização assistida em São Paulo. No presente estudo foram encontrados valores semelhantes, embora a população de São Paulo seja quaro vezes maior do que a de Salvador, os resultados indicam que as pessoas estão mais informadas sobre os tratamentos de RHA e, portanto, buscam com maior frequência um especialista em infertilidade conjugal para realizarem o desejo de formar uma família.

Um estudo de corte transversal conduzido na Grã-Bretanha demonstrou que a taxa de infertilidade foi de 12,5 e 10,1\% em mulheres e homens, respectivamente (DATTA et al., 2016). No Brasil, há poucos estudos que reportem a prevalência de infertilidade. No entanto, a taxa de infertilidade em homens observada no estudo (10,3\%) é semelhante aos dados da GrãBretanha. Por outro lado, a prevalência de infertilidade na mulher $(27,5 \%)$ está acima das taxas observadas em países desenvolvidos como a Grã-Bretanha, mas abaixo da taxa reportada no Sudão (42,8\%) (ELHUSSEIN et al., 2019). Como consequência, é possível que as mulheres inférteis estejam contribuindo diretamente com a redução da taxa de fecundidade, uma vez que a taxa de fecundidade no Brasil (1:9), esta abaixo do esperado (2:1) (CAETANO, 2008; BERQUÓ \& CAVENHAGUI, 2014).

No estudo foi possível observar que os principais fatores associados a infertilidade feminina foram idade avançada, endometriose e obstrução tubária, respectivamente. $\mathrm{O}$ climatério é um fenômeno endócrino decorrente do esgotamento dos folículos ovarianos que ocorre em todas as mulheres de meia idade. Inicia-se entre os 35 e 40 anos, estendendo-se aos 65 anos e caracteriza-se por um estado de hipoestrogenismo progressivo. O envelhecimento feminino pode afetar além da quantidade e qualidade dos oócitos, a função uterina, uma vez que o endométrio perderia sua capacidade de garantir a fixação e o crescimento embrionário (LORENZI; DANELON; SACILOTO, 2005; PESSANHA et al., 2015). Corroborando com estas observações a idade avançada (>35anos) foi o fator mais prevalente como causa de infertilidade entre as mulheres.

Além da idade avançada, ressalta-se a elevada frequência de mulheres com endometriose $(26,5 \%)$ no estudo. Estimativas de prevalência variam até $15 \%$ entre a população em geral (SOUZA et al, 2015) e estudos de larga escala sugerem uma prevalência de até $20 \%$ em mulheres em idade reprodutiva e de 30 a $50 \%$ em mulheres inférteis (SOUZA et al., 2017). Em conjunto, os resultados sugerem que a frequência de endometriose é semelhante aos estudos reportados. No entanto, variações na população de pacientes, métodos e critérios de diagnóstico, e uma falta geral de estudos epidemiológicos bem desenhados, torna-se difícil de determinar valores confiantes para esta doença.

Por último, foi também observado que a obstrução tubária é uma causa frequente entre as mulheres. Um estudo feito por Maior e colaboradores (2007), encontrou uma taxa de 40,9\% das mulheres com obstrução tubária. Infeções por Chlamydia trachomatis e Neisseria gonorrhoeae, trauma cirúrgico, endometriose ou reação a corpos estanhos estão entre as principais causas de lesões e/ou obstrução tubares (QUEIROZ, 2018). 
Nos homens, o estudo mostrou que as principais causas associadas a infertilidade são teratozoospermia e varicocele. Estudo realizado em São Paulo também apresentou a teratozoospermia como uma das maiores causas de infertilidade masculina (PARIZ et al., 2014). Estudo realizado na escola de Medicina da Universidade de Nova Iorque com 495 casais, mostrou que 48,5\% dos pacientes diagnosticados com teratozoospermia isolada apresentam estatisticamente uma menor probabilidade de sucesso na técnica de fertilização quando comparado com sêmen normal de homens férteis (KEEGAN et al., 2007). Em conjunto, os dados sugerem que a teratozoospermia é uma causa frequente entre os homens e que pode influenciar a taxa de fertilização.

A varicocele foi a segunda causa mais frequente de infertilidade masculina no estudo. Um estudo realizado por Wang e colaboradores (2012) demonstrou que a varicocele está presente entre 15 a $23 \%$ dos homens e é a principal causa de infertilidade masculina, chegando a afetar 30 a $40 \%$ dos homens com infertilidade primária, resultado semelhante ao observado no presente estudo. Embora a varicocele afete os parâmetros seminais definidos pela OMS, tais como: azoospermia, oligozoospermia ou astenozoospermia, a doença é considerada como causa reversível mais comum de infertilidade masculina. A correção cirúrgica da varicocele em homens inférteis tem demonstrado melhoras nos parâmetros seminais em 50-80\% dos pacientes (JAROW, 2001).

A qualidade do oócito é um fator chave na fertilidade feminina, refletindo o potencial intrínseco de desenvolvimento do gameta, além de ter um papel crucial não só na fecundação, mas também no desenvolvimento embrionário subsequente (GILCHRIST; LANE; THOMPSON, 2008). Nesse estudo, mais de 50\% dos oócitos puncionados apresentam qualidade oocitária classificada como ruim (158/280), isso pode refletir diretamente na qualidade embrionária, uma vez que dos 310 embriões obtidos, 120 (38,7\%) foram considerados ruins. Oócitos que não apresentam boa qualidade são mais propensos a erros na sua divisão celular, produzindo, como consequência, embriões com alterações genéticas que são impeditivos de gerar uma gravidez saudável, aumentando assim a taxa de abortamento e/ou infertilidade (RIBEIRO, 2013).

Por outro lado, 56,7\% (106/187) dos espermatozoides foram classificados como bons. Este resultado reflete as técnicas de melhoramento seminal (capacitação espermática) e, consequente recuperação dos fatores de motilidade e concentração para utilização da técnica da ICSI. Vale ressaltar que a melhora seminal é relativa ao procedimento feito no laboratório, com o objetivo de adquirir competência fecundante.

Sabe-se que a qualidade embrionária é um fator que também está associada a qualidade do gameta. No entanto, observa-se que a principal causa de má qualidade embrionária nas mulheres foi obstrução tubária, o que representou a causa menos associada ao aumento de oócitos ruins. Por outro lado, nos homens, a qualidade dos embriões e espermatozoide foram oligoastenoteratozoospermia e oligoteratozoopermia. Estes resultados indicam que à qualidade do espermatozoide, ao contrário do oócito, pode comprometer o desenvolvimento embrionário. É necessário estudar cada caso isolado, pois o oócito e o espermatozoide influenciam de forma diferente em cada desenvolvimento embrionário (STEIN, 2010). 


\section{CONCLUSÕES}

O conhecimento dos fatores responsáveis por esta dificuldade reprodutiva tem fundamental importância, pois servem como guia no tratamento e, consequentemente, no aumento das taxas de sucesso. Neste estudo, foi possível observar que entre os casais, a taxa de infertilidade compartilhada por ambos os sexos, onde tanto o homem quanto a mulher possuem infertilidade, foi mais prevalente que a taxa isolada por sexo. A etiologia mais frequente nas mulheres é a idade avançada, seguida pela endometriose. Já entre os indivíduos masculinos, os fatores mais prevalentes foram a teratozoospermia e varicocele, seguidas por alterações diversas alterações seminais.

A técnica de ICSI caracteriza a atual técnica mais complexa de fertilização assistida, auxiliando no tratamento de casais com níveis de infertilidade moderados a graves possibilitando assim a concepção, porém, a maioria dos casais só descobrem que são inférteis de forma tardia, reforçando que o conhecimento da população e até mesmo da comunidade de saúde a respeito da infertilidade e suas causas, ainda é bastante escasso. Logo, estudos como este contribuem não só para que profissionais de saúde estejam mais atentos aos seus pacientes, facilitando possíveis diagnósticos e opções adequadas de tratamento como também possuem grande importância política, uma vez que, conhecendo melhor quais fatores afetam a população, pode-se investir em políticas públicas de saúde que atendam com maior eficácia a população que não tem acesso a clínicas particulares.

\section{REFERÊNCIAS}

BABLOK, L.; DZIADECKI, W.; SZYMUSIK, I.; WOLCZYNSKI, S.; KURZAWA, R.; PAWELCZYK, L.; \& WIELGOS, M. Patterns of infertility in Poland - multicenter study. Neuro Endocrinol Lett, v.32, p.799-804, 2001.

BALABAN, B.; \& URMAN, B. Effect of oocyte morphology on embryo development and implantation. Reproductive biomedicine online, v.12, p.608-15, 2006.

BENTO, M.F.M. A satisfação conjugal e sexual dos casais inférteis: o impacto da infertilidade [tese]. Lisboa: Universidade de Lisboa; 2014.

BERQUÓ, E.S.; \& CAVENHAGUI, S.M. Notas sobre os diferenciais educacionais e econômicos da fecundidade no Brasil. Revista Brasileira de Estudos de População. v.31, n.2, 2014.

BORGES JR, E.; FIGUEIRA, R. C. S.; CORTEZZI, S.S.; MEIRE, M. O. S. Banco de sêmen representa esperança ao paciente oncológico. Rev Bras Reprod Assist, v.12, n.4, p.34-9, 2009.

CAETANO, A. J. Fecundidade abaixo da reposição, população estacionária por migração e efeitos sobre a estrutura etária. Revista Brasileira de Estudos de População, v.25, n.2, p.325-334, 2008.

CONG, J.; LI, P.; ZHENG, L.; \& TAN, J. Prevalence and Risk Factors of Infertility at a Rural Site of Northern China. PloS One, v.11, n.5, p.0155563, 2016. 
COSTA, C.M.V.; TAVARES, R.L.C.; MELO, E.M.; \& MELO, V.H. Infertilidade: causa ou consequência da violência?. Rev Med Minas Gerais, v.26 (SUPL 8), p. 355-61, 2016.

DATTA, J.; PALMER, M.J.; TANTON, C.; GIBSON, L.J.; JONES, K.G.; MACDOWALL, W.; \& JOHNSON, A. M. Prevalence of infertility and help seeking among 15.000 women and men. Human Reproduction, v.31, n.9, p.2108-2118, 2016.

DENTILLO, B.D. Cresce demanda por tratamento de infertilidade, mas o acesso é ainda caro e seletivo. Ciência e Cultura. v.64, n.4, 2012.

DESHPANDE, P.S.; \& GUPTA, A.S. Causes and Prevalence of Factors Causing Infertility in a Public Health Facility. Journal of Human Reproductive Sciences, v.12, n.4, p.287-293, 2019. DOI: 10.4103/jhrs.JHRS_140_18

DURAIRAJANAYAGAM, D. Lifestyle causes of male infertility. Arab Journal of Urology, v.16, n.1, p.10-20, 2018.

ELHUSSEIN, O.G.; AHMED, M.A.; SULIMAN, S.O.; YAHYA, L.I.; \& ADAM, I. Epidemiology of infertility and characteristics of infertile couples requesting assisted reproduction in a low-resource setting in Africa, Sudan. Fertility Research and Practice, v.5, p.7, 2019.

FADHLAOUI, A.; JOLINIÈRE, J.B.L.; \& FEKI, A. Endometriosis and infertility: how and when to treat? Frontiers in surgery, v.1, n.24, 2014.

GEORGE, K.; KAMATH, M.S. Fertility and age. Journal of Human Reproductive Sciences, v.3, n.3, p.121-123, 2010.

GILCHRIST, R.B.; LANE, M.; \& THOMPSON, J.G. Oocyte-secreted factors: regulators of cumulus cell function and oocyte quality. Human reproduction update, v.14, p.159-77, 2008 .

GRADVOHL, S.M.O.; OSIS, M.J.D.; \& MAKUCH, M.Y. Stress of men and women seeking treatment for infertility. Revista brasileira de ginecologia e obstetricia: revista da Federacao Brasileira das Sociedades de Ginecologia e Obstetricia. v.35, n.6, p.255-61, 2013.

GRANJEIRO, C.H.P. Avaliação genômica da infertilidade masculina idiopática por azoospermia não obstrutiva [Tese]. Ribeirão Preto: Universidade de São Paulo; 2018.

HARRIS, I.D.; FRONCZAK, C.; ROTH, L.; MEACHAM, R.B. Fertility and the aging male. Reviews in Urology, v.13, n.4, p.184-190, 2011.

HOCHSCHILD, F.; ADAMSON, G.D.; DE MOUZON, J.; ISHIHARA, O.; MANSOUR, R.; NYGREN, K.; \& VAN DER POEL, S. International Committee for Monitoring Assisted Reproductive Technology (ICMART) and the World Health Organization (WHO) revised glossary of ART terminology, 2009. Human reproduction, v.92, n.5, p.1520-4, 2009.

ILACQUA, A.; IZZO, G.; EMERENZIANI, G.P.; BALDARI, C.; AVERSA, A. Lifestyle and fertility: the influence of stress and quality of life on male fertility. Reproductive biology and endocrinology: RB\&E, v.16, n.1, p.1-11, 2018. 
JAROW, J.P. Effects of varicocele on male fertility. Human Reproduction Update, v.7, n.1, p.59-64, 2001.

KEEGAN, B.R.; BARTON, S.; SANCHEZ, X.; BERKELEY, A.S.; KREY, L.C.; \& GRIFO, $\mathrm{J}$. Isolated teratozoospermia does not affect in vitro fertilization outcome and is not an indication for intracytoplasmic sperm injection. Fertility and sterility, v.88, n.6, p.1583-88, 2007.

LEITE, R.R.Q.; \& FROTA, A.M.M.C. O desejo de ser mãe e a barreira da infertilidade: uma compreensão fenomenológica. Revista da Abordagem Gestáltica: Phenomenological Studies, v.20, n.2, p.151-160, 2014.

LI, W.N.; JIA, M.M.; PENG, Y.Q.; DING, R.; FAN, L.Q.; \& LIU, G. Semen quality pattern and age threshold: a retrospective cross-sectional study of 71,623 infertile men in China, between 2011 and 2017. Reproductive Biology and Endocrinology, v.17, n.107, 2019.

LORENZI, D.R.S.; DANELON, C.; \& SACILOTO, B.; \& PADILHA JR, I. Fatores indicadores da sintomatologia climatérica. Revista Brasileira de Ginecologia e Obstetrícia, v.27, n.1, p.7-11, 2005.

MAIOR, M.D.C.F.S.; SOUZA, A.I.D.; AMORIM, M.M.R.D.; VALENTE, E.P.; COSTA, A. R.; CUNHA, A.S.C.D.; \& CABRAL-FILHO, J. E. Achados de laparoscopias ginecológicas realizadas em mulheres com dificuldade reprodutiva atendidas em um hospital-escola: série de casos. Revista Brasileira de Ginecologia e Obstetrícia, v.29, n.6, p.297-302, 2007.

OMS. Organização Mundial da Saúde. CID 10: Classificação estatística internacional de doenças e problemas relacionados à saúde, 2008.

PARIZ, J.R.; CHEMES, H.E.; MONTEIRO, R.A.C; PIERI, P.C; \& HALLAK, J. Aplicação da avaliação ultraestrutural de espermatozoides na rotina da andrologia. Reprodução \& Climatério, v.26, n.1, p.37-40, 2014.

PESSANHA, J.C.; MOORE, A.C.S.; PEREIRA, I.; \& ESPARRACHIARI, L.C.C. Repercussões da endometriose na vida de mulheres desde os primeiros sintomas até o diagnóstico. Perspectivas Online: Biológicas \& Saúde, v.5, n.18, 2015.

QUEIROZ, C. Gestações transnacionais. Revista Pesquisa Fapesp, p.70-75, 2018.

RIBEIRO, R.S.C. Estudo de produtos de abortamento de constituição cromossómica aneuplóide: avaliação da expressão dos genes MAD2L2, BUB1, BUB1B e KIF2C [tese]. Porto: Universidade Porto; 2013.

SOUZA, G.K.T.; COSTA, J.R.G.C.; OLIVEIRA, L.L.; \& LIMA, L.R. Endometriose x infertilidade: revisão de literatura. Encontro de Extensão, Docência e Iniciação Científica (EEDIC), v.3, n.1, p.1-4, 2017.

SOUZA, T. R.; QUEIROZ, A. P.; ASSUMPÇAO, B. R.; \& FLORES S. F. Prevalência dos sintomas da endometriose: Revisão Sistemática. Revista CES Medicina, v.29, n.2 p.211-26, 2015.

Persp. online: biol. \& saúde, Campos dos Goytacazes, 33 (10) 1-14, 2020 seer.perspectivasonline.com.br 
STEIN, A.C. Avaliação dos parâmetros seminais no desenvolvimento embrionário em mulheres submetidas à fertilização in vitro com estimulação ovariana [tese]. Porto Alegre: Universidade Federal do Rio Grande do Sul; 2010.

WANG，Y.J.; ZHANG， R.Q.; LIN，Y.J.; ZHANG，R.G.; \& ZHANG，W.L. Relationship between varicocele and sperm DNA damage and the effect of varicocele repair: a metaanalysis. Reproductive biomedicine online, v.25, n.3, p.307-14, 2012. 